\title{
Controle químico e caracterização da superfície foliar de Conyza bonariensis e C. canadensis (Asteraceae) ${ }^{1}$
}

\section{Chemical control and leaf surface characterization of Conyza bonariensis and $C$. canadensis (Asteraceae)}

\author{
Estela Maris Inacio $^{2}$; Patricia Andrea Monquero ${ }^{3}$
}

Resumo - O gênero Conyza inclui aproximadamente 60 espécies de plantas distribuídas em quase todo o mundo. As espécies Conyza canadensis e C. bonariensis destacam-se por infestarem áreas abandonadas, pastagens, culturas perenes e lavouras anuais. Os objetivos desse trabalho foram: (1) caracterizar a superfície foliar de $C$. bonariensis e $C$. canadensis através da microscopia eletrônica de varredura; (2) determinar o controle destas plantas com o uso do herbicida glyphosate em associação com herbicidas de distintos mecanismos de ação. Para analisar a superfície foliar foram retirados dois segmentos de aproximadamente $50 \mathrm{~mm}^{2}$ da região mediana das folhas jovens de $C$. bonariensis e $C$. canadensis, porém totalmente expandidas para análise em microscópio eletrônico de varredura. No estudo de controle químico foi utilizado o delineamento experimental inteiramente casualizado com quatro repetições, os herbicidas foram aplicados em pós-emergência com as plantas apresentando de três a quatro pares de folhas verdadeiras. Os tratamentos utilizados foram glyphosate $\left(0,42 \mathrm{~kg} \mathrm{ha}^{-1}\right)$, em associação com amônio - glufosinato $\left(0,50 \mathrm{~kg} \mathrm{ha}^{-1}\right), 2,4-\mathrm{D}\left(1,0 \mathrm{~kg} \mathrm{ha}^{-1}\right)$, bentazon $\left(0,72 \mathrm{~kg} \mathrm{ha}^{-1}\right)$, chlorimuronethyl $\left(0,15 \mathrm{~kg} \mathrm{ha}^{-1}\right)$, carfentrazone-ethyl $\left(0,03 \mathrm{~kg} \mathrm{ha}^{-1}\right)$, metribuzin $\left(0,48 \mathrm{~kg} \mathrm{ha}^{-1}\right)$ e sulfentrazone $\left(0,60 \mathrm{~kg} \mathrm{ha}^{-1}\right)$, além dos mesmos herbicidas aplicados isoladamente e um tratamento testemunha. Ambas as espécies são anfiestomáticas, apresentam estômatos na superfície adaxial e abaxial, possuem tricomas tectores unicelulares e pluricelulares, sendo que a espécie C. bonariensis possui visualmente maior quantidade de tricomas na face adaxial. Para $C$. bonariensis, os tratamentos de amônio-glufosinato e a associação de glyphosate com amônio-glufosinato, bentazon, chlorimuron-ethyl e metribuzin foram os mais efetivos no controle. Para C. canadensis os tratamentos mais eficientes no controle foram: glyphosate + amônio-glufosinato, glyphosate + bentazon, e glyphosate + metribuzin.

Palavras-chaves: herbicidas, anatomia foliar, glyphosate, mecanismo de ação

Abstract - The genus Conyza includes approximately 60 species which are widely distributed across the globe. The species Conyza canadensis and Conyza bonariensis are noted for their ability to invade abandoned areas, pastures, perennial crops and annual crops. The objectives of the present study were to: (1) characterize the leaf surface of $C$. bonariensis and $C$. canadensis by scanning electron microscopy, (2) evaluate the control of these plants with glyphosate mixed with

\footnotetext{
${ }^{1}$ Recebido para publicação em 03/12/2013 e aceito em 01/02/2014.

${ }^{2}$ Doutorada no Programa de Pós-Graduação em Fitotecnia da Universidade de São Paulo (USP), Piracicaba, SP. Email: <estelainacio@hotmail.com>.

${ }^{3}$ Docente do Departamento De Recursos Naturais e Proteção Ambiental do Centro de Ciências Agrárias da Universidade Federal de São Carlos, São Carlos, SP, Brasil. Email: pamonque@cca.ufscar.br.
} 
herbicides distinct mechanisms of action. To analyze the leaf surface by scanning electron microscopy two segments of approximately $50 \mathrm{~mm}^{2}$ were taken from the median region of young (but fully expanded) leaves of $C$. bonariensis and $C$. Canadensis. The herbicide control study was conducted in completely randomized design with four replicates. Herbicides were applied post-emergence when plants showed three to four pairs of true leaves. The herbicide treatments were: glyphosate $\left(0.42 \mathrm{~kg} \mathrm{ha}^{-1}\right)$, mixed with glufosinate-ammonium $\left(0.5 \mathrm{~kg} \mathrm{ha}^{-1}\right), 2,4-\mathrm{D}(1.0 \mathrm{~kg}$ $\left.\mathrm{ha}^{-1}\right)$, bentazon $(0.72 \mathrm{~kg} \mathrm{ha-1})$, chlorimuron-ethyl $\left(0.15 \mathrm{~kg} \mathrm{ha}^{-1}\right)$, carfentrazone-ethyl $\left(0.03 \mathrm{~kg} \mathrm{ha}^{-}\right.$ $\left.{ }^{1}\right)$, metribuzin $\left(0.48 \mathrm{~kg} \mathrm{ha}^{-1}\right)$ and sulfentrazone $\left(0.6 \mathrm{~kg} \mathrm{ha}^{-1}\right)$, as well as the same herbicides applied in isolation and a control treatment without herbicide. Both species are amphistomatic (with stomata on the adaxial and abaxial surface), and both possess unicellular and multicellular non-gladular trichomes. $C$. bonariensis had a visually larger number of trichomes on the adaxial leaf surface. For $C$. bonariensis the following treatments were more effective than the control: glufosinate-ammonium and glyphosate mixed with glufosinate-ammonium, bentazon, chlorimuron-ethyl and metribuzin. For $C$. canadensis the treatments more effective than the control were: glyphosate + glufosinate-ammonium, glyphosate + bentazon, and glyphosate + metribuzin.

Keywords: herbicides, leaf anatomy, glyphosate, mechanism of action

\section{Introdução}

Atualmente entre as principais plantas daninhas existentes no Brasil existe uma atenção especial com relação à Conyza bonariensis e $C$. canadensis, que são espécies da família Asteraceae, originadas da América do Sul e do Norte respectivamente.

No Brasil, as duas espécies são denominadas popularmente de "buva" ou "voadeira" e têm distribuição comum entre as regiões Centro Oeste e Sul, infestando áreas cultivadas com citros, café, pastagens e lavouras anuais, como a cultura da soja (Yamauti et al., 2010). A buva é uma planta anual, que germina no outono/inverno encerrando seu ciclo durante o verão, portanto, é classificada com ciclo de vida de inverno e verão (Kissmann \& Groth, 1999)

O controle de $C$. bonariensis e $C$. canadensis em lavouras de culturas anuais (cereais de inverno, soja e milho) é feito com emprego de herbicidas específicos ou nãoseletivos. Em trigo, $C$. bonariensis e $C$. canadensis é controlada com 2,4-D e chlorimuron-ethyl e metsulfuron-methyl. Nas culturas de soja e milho o controle de $C$. bonariensis e $C$. canadensis é realizado principalmente com uso de glyphosate na dessecação e pré-semeadura dessas culturas (Vidal et al., 2007).

Entretanto, em algumas regiões do Brasil, foram identificadas biótipos resistentes ao glyphosate de $C$. bonariensis $e \quad C$. canadensis em pomares de citros (Moreira et al., 2007) e em áreas de soja transgênica tolerante ao glyphosate (Vargas et al., 2007; Lamego \& Vidal, 2008).

Segundo Lazarato et al. (2008), é frequente a ocorrência de ambas as espécies de C. bonariensis e $C$. canadensis associadas, as quais apresentam adaptabilidade ecológica em sistemas conservacionistas. No entanto, é comum haver confusão na diferenciação das espécies.

Portanto, a identificação correta das mesmas são fatores importantes para que se possa escolher apropriadamente a melhor estratégia de controle, uma vez que as características genéticas entre as espécies podem influenciar suas respostas aos herbicidas (Vargas et al., 2007).

O presente trabalho teve como objetivos a caracterização foliar de $C$. bonariensis e $C$. canadensis e avaliação do controle destas plantas daninhas, utilizando diversos herbicidas 
aplicados isoladamente ou em mistura com glyphosate.

\section{Material e Métodos}

\section{Caracterização foliar de $C$. canadensis e $C$. bonariensis}

Para análise em microscópio eletrônico de varredura, dois segmentos de aproximadamente $50 \mathrm{~mm}$ quadrados foram retirados da região mediana das folhas jovens $\mathrm{e}$ totalmente expandidas de $C$. bonariensis e $C$. canadensis para análise das superfícies adaxial e abaxial. A primeira observação em microscópio eletrônico de varredura foi realizada em setembro/2010 com plantas jovens, apresentando três a quatro pares de folhas verdadeiras. A segunda observação foi realizada em dezembro/2010 com plantas adultas, apresentando mais de oito pares de folhas verdadeiras. O preparo das amostras foi realizado no Laboratório de Microscopia Eletrônica de Varredura, pertencente à Escola Superior de Agricultura Luiz de Queiroz (Esalq / USP). As imagens foram processadas no microscópio eletrônico de varredura pertencente ao Instituto de Biologia / Unicamp.

O preparo da amostra foi realizado seguindo a metodologia padrão para preparo de amostras utilizados no laboratório Núcleo de Apoio a Pesquisa em Microscopia Eletrônica aplicada a Pesquisa Agropecuária (Tanaka \& Kitajima 2009), da Escola Superior de Agricultura "Luiz de Queiroz" - USP.

Os segmentos vegetais foram fixados utilizando o Fixador Karnovsky modificado (glutaraldeído 2,5\%, formaldeído 2,5\% em tampão cacodilato de sódio $0,05 \mathrm{M}, \mathrm{pH} 7,2$, $\mathrm{CaCl}_{2}$ 0,001 M.). As amostras foram colocadas em tubos eppendorff de $1,5 \mathrm{~mL}$ e mantidas nessa solução durante 12 horas na geladeira. $\mathrm{O}$ volume do fixador foi de cerca de 10 vezes o da amostra. Após esse período as amostras foram submetidas a soluções de concentrações crescentes de acetona $\mathrm{C}_{3} \mathrm{H}_{6} \mathrm{O}(30,50,70,90$ e $100 \%)$, permanecendo cerca de 10 minutos em cada uma, sendo que esse processo foi repetido três vezes na solução de $100 \%$. A seguir, as amostras foram colocadas em gaiolas individuais devidamente identificadas e levadas para aparelho CPO 050 da Balzers, onde passaram pelo processo de secagem ao ponto crítico, que consiste em uma câmara hermeticamente fechada que permite a entrada de $\mathrm{CO}_{2}$, que se liquefaz, ficando a amostra nele mergulhada. Esta câmara foi aquecida até 40 ${ }^{\circ} \mathrm{C}$, quando então, gás é lentamente eliminado e as amostras ficam secas.

As amostras secas foram então montadas em stubs e levadas para o metalizador MED 010 da Balzers, onde foram recobertas com uma fina camada de ouro. Após o preparo, as amostras foram levadas para observação em microscópio eletrônico de varredura JEOL, pertencente à Universidade de Campinas (Unicamp - Instituto de Biologia Laboratório de Microscopia Eletrônica), onde foram digitalizadas as imagens mais nítidas.

\section{Controle químico de $C$. canadensis e $C$. bonariensis}

O experimento foi conduzido em casade-vegetação do Centro de Ciências Agrárias, Universidade Federal de São Carlos, localizada no município de Araras/SP, localizada a $22^{\circ} 18^{\prime} 25^{\prime \prime}$ de latitude Sul e $47^{\circ} 18^{\prime} 03^{\prime \prime}$ de longitude Oeste, altitude de $611 \mathrm{~m}$, onde o clima na região apresenta verões quentes e úmidos e invernos secos.

As sementes das espécies de $C$. bonariensis e $C$. canadensis foram adquiridas da empresa Cosmos Agrícola Produção e Serviços Ltda, coletadas de áreas citrícolas da região de Matão/SP, com suspeita de resistência ao glyphosate.

As sementes foram colocadas para germinar em caixas plásticas preenchidas com substrato composto por casca de pinus bioestabilizada, turfa vegetal, vermiculita expandida, fibra de coco, corretivos de acidez, adubo super fostato simples + NPK + micronutrientes. As bandejas foram mantidas 
irrigadas até as plantas apresentarem um par de folhas verdadeiras, sendo depois transplantadas para vasos com capacidade de $0,4 \mathrm{~L}$, também preenchidos com o mesmo substrato para horticultura, obtendo três plantas por vaso. O delineamento experimental utilizado foi inteiramente casualizado, constituído de 15 tratamentos, sendo um tratamento testemunha, sem aplicação de herbicida e quatro repetições. Os tratamentos podem ser observados na Tabela 1.

Tabela 1. Tratamentos aplicados nas plantas de $C$. bonariensis e $C$. canadensis.

\begin{tabular}{lcc}
\hline Tratamento & $\mathrm{kg} \mathrm{ou} \mathrm{L} \mathrm{i.a.} \mathrm{ha} \mathrm{h}^{-1}$ & $\mathrm{~kg}$ ou L do p.c. ha \\
\hline Testemunha & - & - \\
Glyphosate & 0,42 & 2,0 \\
Amônio-glufosinato* & 0,5 & 2,0 \\
Bentazon* & 0,72 & 1,2 \\
Carfentrazone-ethyl* & 0,03 & 0,075 \\
Chlorimuron-ethyl* & 0,15 & 0,040 \\
Metribuzin & 0,48 & 1,0 \\
Sulfentrazone & 0,6 & 1,5 \\
Glyphosate + amônio-glufosinato* & $0,42+0,5$ & $2,0+2,0$ \\
Glyphosate + bentazon* & $0,42+0,72$ & $2,0+1,2$ \\
Glyphosate + carfentrazone-ethyl* & $0,42+0,03$ & $2,0+0,075$ \\
Glyphosate + chlorimuron-ethyl* & $0,42+0,15$ & $2,0+0,040$ \\
Glyphosate + metribuzin & $0,42+0,48$ & $2,0+1,0$ \\
Glyphosate + sulfentrazone & $0,42+0,6$ & $2,0+1,2$ \\
Glyphosate + 2,4-D & $0,42+1,0$ & $2,0+1,5$ \\
\hline
\end{tabular}

* Uso de adjuvante Gotafix na calda $(0,1 \%$ v/v); i.a. $=$ ingrediente ativo, p.c. $=$ produto comercial.

Quando as plantas apresentavam entre 4 e 6 pares de folhas verdadeiras, foram aplicados os tratamentos herbicidas em pósemergência, utilizando-se um pulverizador costal de pressão constante, pressurizado por $\mathrm{CO}_{2}$, com pontas do tipo leque XR 110.02, pressão de $2,0 \mathrm{kgf} \mathrm{cm}^{-2}$, volume de calda de $200 \mathrm{~L} \mathrm{ha}^{-1}$. Durante a aplicação dos herbicidas a umidade relativa do ar foi de $40 \%$ e temperatura de $29,9^{\circ} \mathrm{C} . \mathrm{g}$

As plantas daninhas foram avaliadas aos 7, 14, 28 e 35 dias após o tratamento (DAT), utilizando uma escala de $0 \%$, representando efeito nulo dos herbicidas sobre as plantas, a $100 \%$, que representa a morte total das plantas (ALAM, 1974). Aos 42 DAT as plantas foram cortadas e levadas para estufa com temperatura constante de $60^{\circ} \mathrm{C}$ durante 48 horas. A porcentagem de produção da massa seca da parte aérea foi comparada com a testemunha, sem aplicação de herbicida.

Os dados obtidos foram submetidos a analise de variância pelo teste $\mathrm{F}$ e as médias comparadas pelo teste de Tukey, ao nível de $5 \%$ de significância.

\section{Resultados e Discussão}

\section{Caracterização foliar de $C$. canadensis e $C$. bonariensis}

A superfície adaxial de $C$. bonariensis e C. canadensis são morfologicamente semelhantes, apresentando tricomas tectores, que são de dois tipos: longos com extremidade afilada e curtos com extremidade curva, inclusive nas margens das folhas (Figuras 1 a 4). Os tricomas são estruturas importantes, pois mantêm uma atmosfera saturada em vapor de água em torno da folha, reduzindo assim, a transpiração, além de regular a temperatura e a reflexão da radiação solar que atinge a superfície das plantas (Larcher, 2004). Além da importância fisiológica, os tricomas presentes na superfície foliar podem interceptar gotas pulverizadas, impedindo que estas alcancem a epiderme propriamente dita (Procópio et al, 2003a). 


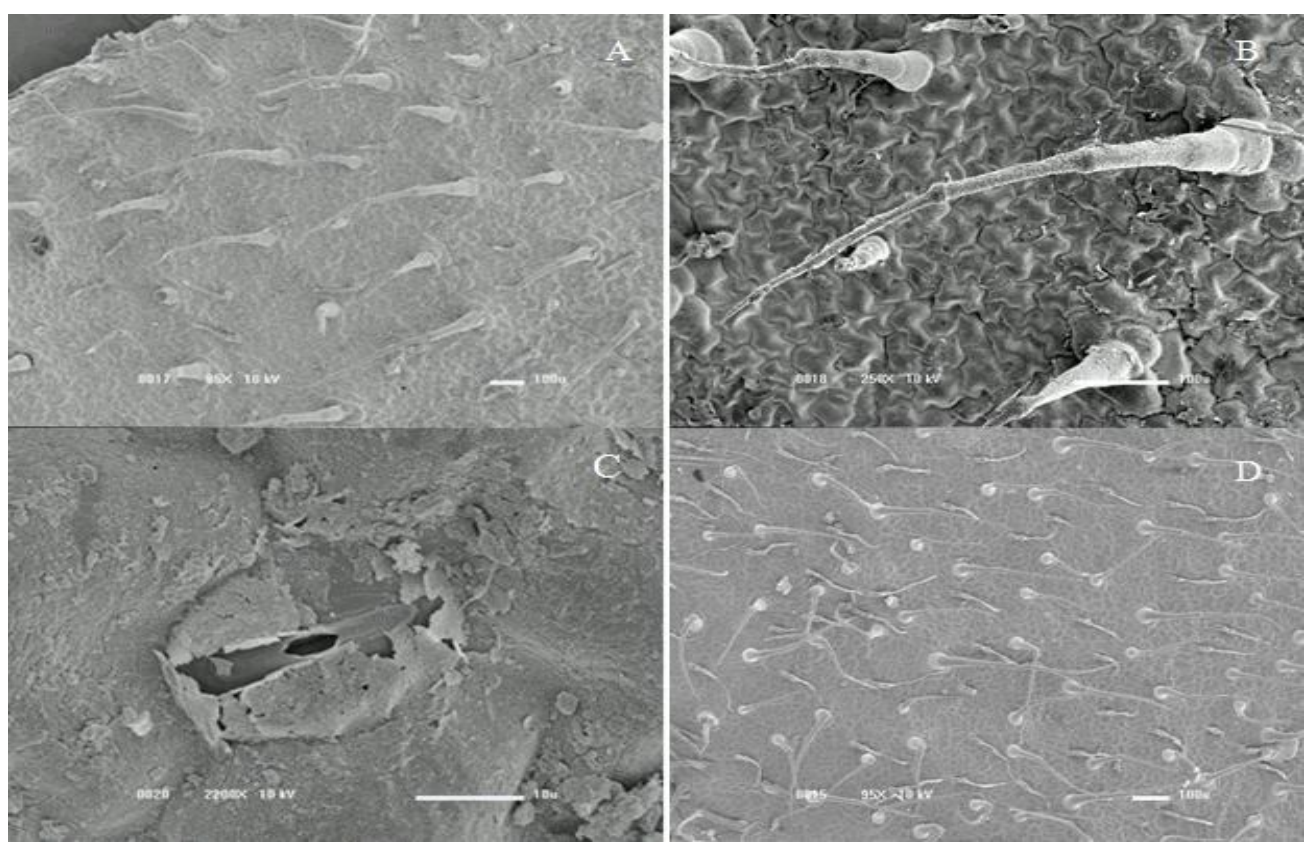

Figura 1. Superfície adaxial de C. canadensis: vista geral dos tricomas tectores unicelulares e pluricelulares com aumento de $85 \mathrm{X}$ (Imagem A), detalhes de tricomas tectores pluricelulares com aumento de 250X (Imagem B), detalhe de estômato anomocítico utilizando aumento de 2200X (Imagem C), vista geral da superfície foliar e de tricomas tectores com aumento de 95X (Imagem D).

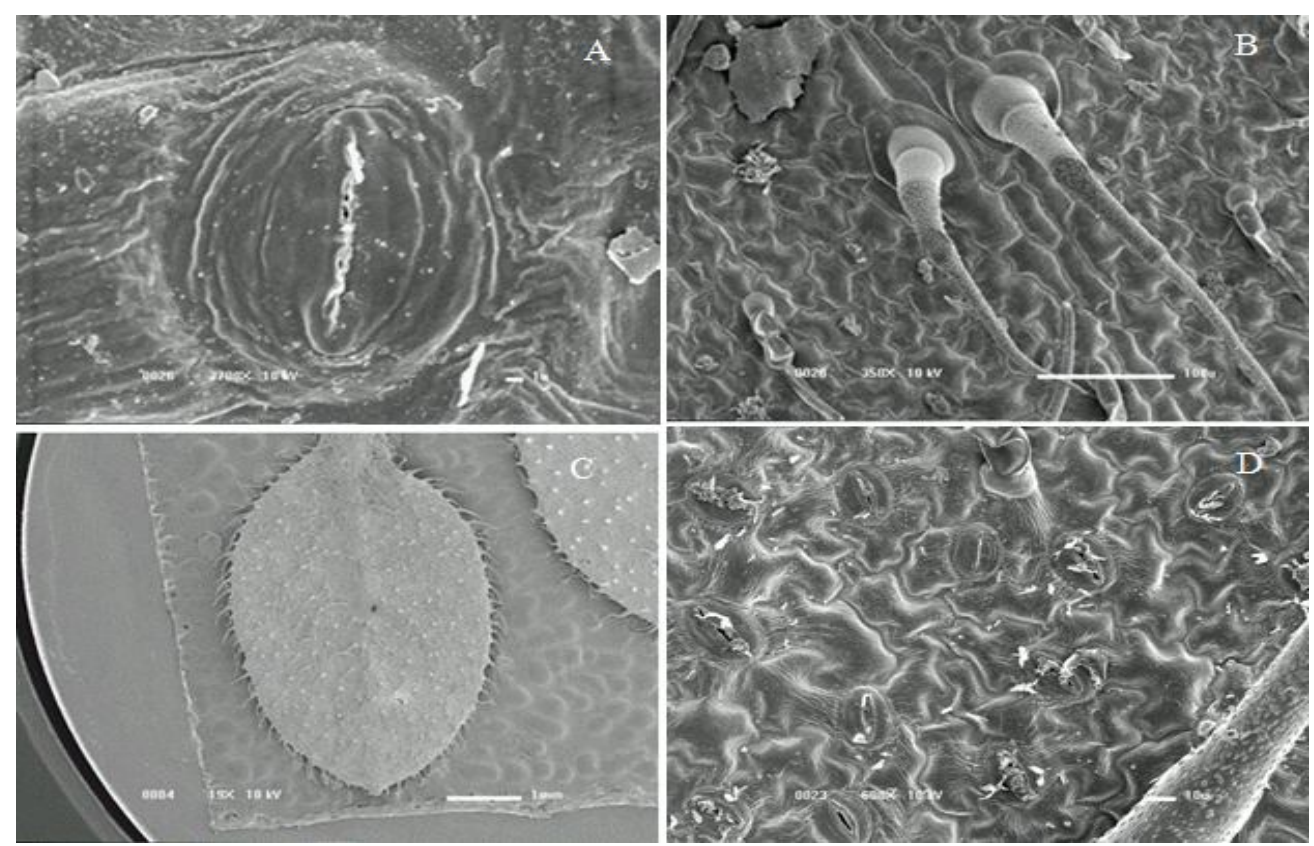

Figura 2. Superfície abaxial de C. canadensis: detalhe de estômato anomocítico utilizando aumento de 3700X (Imagem A), detalhe dos tricomas tectores pluricelulares com aumento de 350X (Imagem B), vista geral da superfície foliar enfatizando a quantidade de tricomas tectores utilizando aumento de 150X (Imagem C), vista geral dos estômatos anomocíticos através do aumento de 600X (Imagem D). 


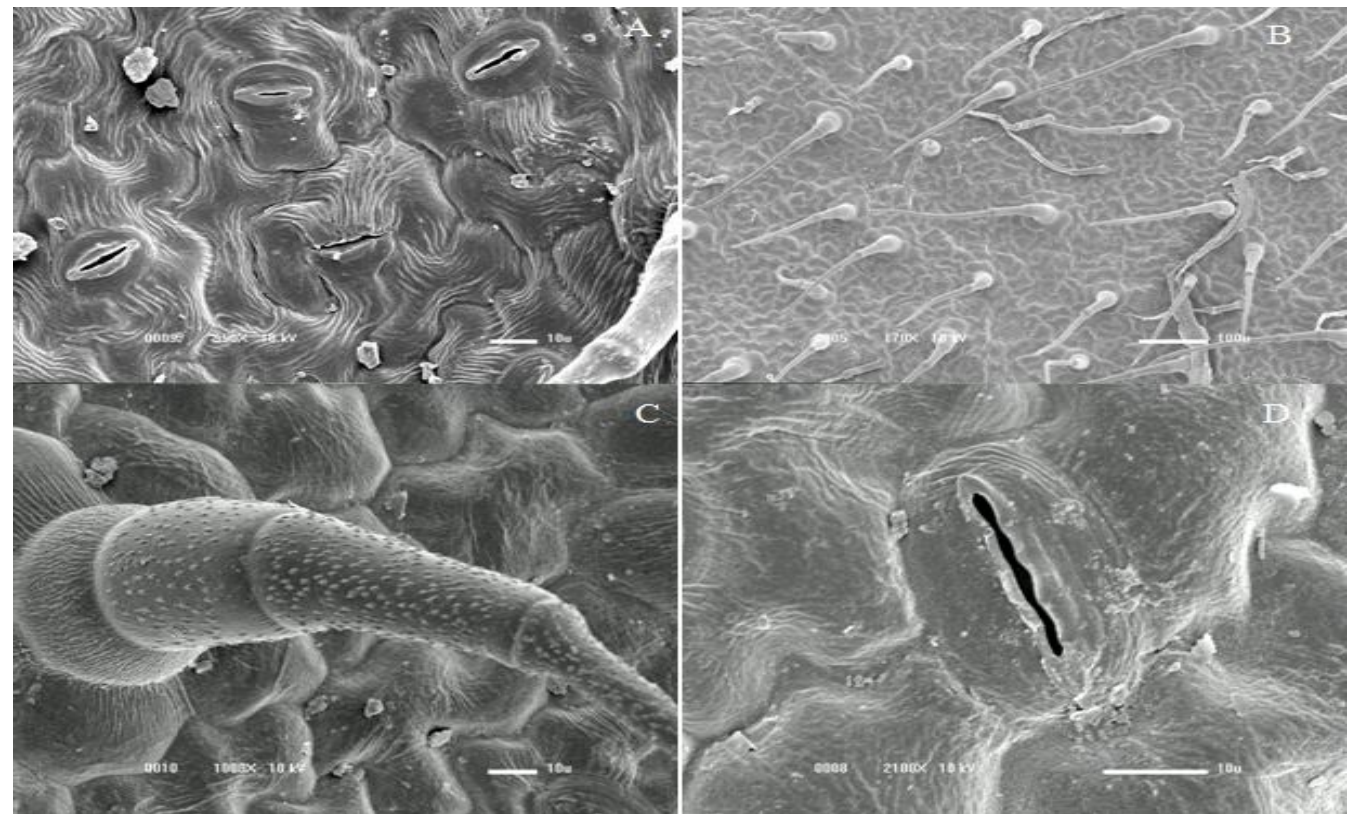

Figura 3. Superfície adaxial de $C$. bonariensis: vista geral dos estômatos anomocíticos e da superfície foliar utilizando aumento de 950X (Imagem A), vista geral da superfície foliar enfatizando a quantidade de tricomas tectores unicelulares e pluricelulares utilizando aumento de 170X (Imagem B), detalhe de tricoma tector pluricelular com aumento de 1000X (Imagem C), detalhe de um estômato anomocítico utilizando aumento de 2100X (Imagem D).
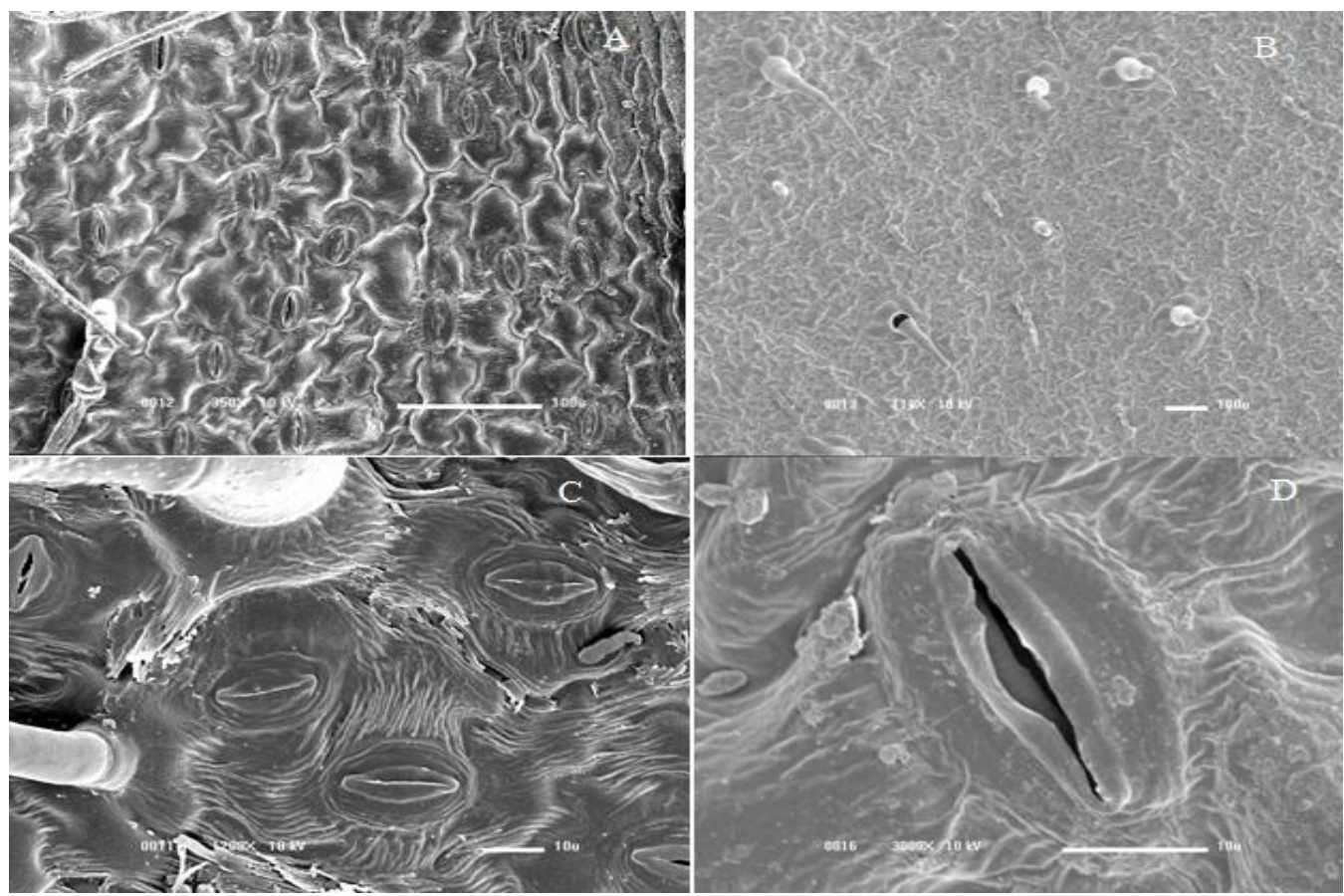

Figura 4. Superfície abaxial de $C$. bonariensis: vista geral dos estômatos anomocíticos e da superfície foliar utilizando aumento de 350X (Imagem A), com vista geral da superfície foliar enfatizando a quantidade de tricomas através do aumento de 110X (Imagem B), detalhe de estômatos anomocíticos com aumento de 1200X (Imagem C), detalhe de um estômato anomocítico utilizando aumento de 3000X (Imagem D). 
Com relação à presença de estômatos, C. bonariensis e C. canadensis apresentaram características anfiestomáticas, ou seja, com estômatos em ambas as faces das folhas. Os estômatos de ambas as espécies são anomocíticos, ou seja, não apresentam células subsidiárias diferenciadas (Appezzato-DaGlória \& Guerreiro, 2003). A absorção estomática possui limitada importância prática, uma vez que, para ocorrer movimento do herbicida através do ostíolo aberto para dentro da câmara estomática e deste para o citoplasma das células, seria necessária diminuição drástica da tensão superficial da gota pulverizada. Essa redução somente seria possível com o uso de surfactantes organosiliconados que além de serem caros, são incompatíveis com uma série de herbicidas. Oliveira Jr. \& Bacarim (2001) concluíram, que a penetração estomática de gotas pulverizadas a partir de uma solução baseada em água, mesmo com a adição de surfactantes, é provavelmente um processo de menor importância em aplicações de herbicidas no campo. Outro fator que leva os pesquisadores a acreditar na pouca importância da absorção de herbicidas através dos estômatos é o fato de estes, em vários horários do dia, estarem fechados, inclusive em aplicações noturnas (Ferreira et al., 2002).

Não foi detectada a presença de cristais de ceras epicuticulares em ambas as faces da superfície foliar das espécies estudadas. A ausência de cristais de ceras epicuticulares também foi observada em outras espécies de plantas, como Vitis vinifera, Beta vulgares e Trifolium repens e Ipomoea grandifolia. A presença de ceras amorfas pode estar associada à baixa quantidade de ceras na superfície foliar ou à predominância de álcool primário na composição química das ceras (Baker \& Bukovac, 1971; Monquero, 2003).

$\mathrm{Na}$ aplicação de defensivos, Bukovac \& Petracek (1993) acreditam que a penetração foliar começa quando a solução é retida pela superfície da planta, sendo um processo continuo composto de três etapas: sorção, difusão e dessorção. A sorção consiste na distribuição do ingrediente ativo entre a solução aquosa de pulverização e a cutícula. $\mathrm{O}$ soluto é então difundido através da cutícula e, quando as moléculas chegam na interface da superfície cuticular, são dessorvidas da cutícula no apoplasto aquoso.

\section{Controle químico de $C$. canadensis e $C$. bonariensis}

Para o controle de C. bonariensis, aos 35 DAT, os tratamentos com amônioglufosinato isolado e as associações de glyphosate com amônio-glufosinato, bentazon, chlorimuron-ethyl e metribuzin foram as mais efetivos (Tabela 2). O herbicida glyphosate isolado não foi eficiente no controle dessa espécie em nenhuma das avaliações. No trabalho realizado por Paula et al. (2011), foi observado maior controle desta espécie com uso de glyphosate $\left(1.080 \mathrm{~g} \mathrm{ha}^{-1}\right)+2,4-\mathrm{D}(1209$ $\left.\mathrm{g} \mathrm{ha}^{-1}\right)$, seguido de glyphosate $\left(1.080 \mathrm{~g} \mathrm{ha}^{-1}\right)+$ chlorimuron-ethyl $\left(32 \mathrm{~g} \mathrm{ha}^{-1}\right)$, ocorrendo maior eficiência no controle de $C$. bonariensis com uso de associações de herbicidas, quando comparado ao tratamento isolado com glyphosate. Em outro trabalho, o uso isolado de glyphosate controlou $54 \%$ das plantas de $C$. bonariensis, já quando aplicado sequencialmente com 2,4-D houve melhora significativa do controle para $85-93 \%$ (Werth et al., 2010).

O herbicida amônio-glufosinato aplicado isoladamente foi capaz de controlar $86 \%$ da C. bonariensis a partir dos 14 DAT, sendo que aos 28 DAT as plantas apresentaram leves sinais de recuperação, mas não havendo evolução, uma vez que aos 35 DAT estas apresentaram 72,5\% de controle (Tabela 2).

$\mathrm{O}$ herbicida bentazon isolado não controlou satisfatoriamente a $C$. bonariensis em nenhuma das avaliações. No início das avaliações observaram-se alguns sintomas de fitotoxicidade, como amarelecimento ou mesmo necrose das folhas, mas completada a desativação do produto, o desenvolvimento 
voltou a ser normal, sem efeito negativo sobre essa planta daninha.

Já o herbicida carfentrazone-ethyl aplicado isoladamente e a sua interação com glyphosate foi eficiente somente aos 7 DAT. Entretanto, após esse período, as plantas se recuperaram sendo que aos 35 DAT, embora plantas estivessem menores, observou-se leve sintoma de clorose.

O herbicida chlorimuron-ethyl aplicado isoladamente não controlou a espécie em nenhuma das avaliações. Já a interação entre glyphosate + chlorimuron o controle foi efetivo a partir dos 28 aos 35 DAT, obtendo-se $82 \%$. Em trabalho realizado por Monquero et al. (2001), observaram que a adição de chlorimurom - etílico ao glyphosate promoveu efeito sinergístico no controle de Amaranthus hybridus e Richardia brasiliensis.

O metribuzin isolado não controlou as plantas, uma vez que o controle foi de $25 \%$ aos 35 DAT. Entretanto, a mistura em tanque entre metribuzin + glyphosate foi eficiente no controle a partir dos 7 DAT, obtendo $97,50 \%$ e aos 14 DAT ocorrendo a morte das plantas.

Tabela 2. Porcentagem de controle de $C$. bonariensis aos 7, 14, 28 e 35 DAT.

\begin{tabular}{|c|c|c|c|c|c|}
\hline \multirow{3}{*}{ Tratamentos } & \multirow{3}{*}{$\begin{array}{l}\text { Dose i.a. } \\
\mathrm{kg} \mathrm{ha}^{-1}\end{array}$} & \multicolumn{4}{|c|}{ DAT } \\
\hline & & 7 & 14 & 28 & 35 \\
\hline & & \multicolumn{4}{|c|}{$\%$} \\
\hline Testemunha & 0,00 & $0,00 \mathrm{e}$ & $0,00 \mathrm{e}$ & $0,00 \mathrm{~d}$ & $0,00 \mathrm{~d}$ \\
\hline Glyphosate & 0,42 & $3,75 \mathrm{de}$ & $8,75 \mathrm{de}$ & $0,00 \mathrm{~d}$ & $5,00 \mathrm{~d}$ \\
\hline Amônio-glufosinato & 0,50 & $73,75 \mathrm{~b}$ & $86,25 \mathrm{a}$ & $67,50 \mathrm{~b}$ & $72,50 \mathrm{~b}$ \\
\hline Bentazon & 0,72 & $18,75 \mathrm{~cd}$ & $3,75 \mathrm{de}$ & $7,50 \mathrm{~cd}$ & $16,25 \mathrm{~cd}$ \\
\hline Carfentrazone-ethyl & 0,03 & $82,50 \mathrm{ab}$ & $17,50 \mathrm{~cd}$ & $0,00 \mathrm{~d}$ & $5,00 \mathrm{~d}$ \\
\hline Chlorimuron-ethyl & 0,15 & $5,00 \mathrm{de}$ & $1,25 \mathrm{de}$ & $2,50 \mathrm{~d}$ & $28,75 \mathrm{c}$ \\
\hline Metribuzin & 0,48 & $10,00 \mathrm{~d}$ & $0,75 \mathrm{e}$ & $8,75 \mathrm{~cd}$ & $25,00 \mathrm{c}$ \\
\hline Sulfentrazone & 0,6 & $35,00 \mathrm{c}$ & $7,50 \mathrm{~cd}$ & $6,25 \mathrm{~cd}$ & $23,75 \mathrm{c}$ \\
\hline Glyphosate + amônio-glufosinato & $0,42+0,5$ & $86,25 \mathrm{a}$ & $98,75 \mathrm{a}$ & $90,00 \mathrm{a}$ & $92,50 \mathrm{a}$ \\
\hline Glyphosate + bentazon & $0,42+0,72$ & $94,75 \mathrm{a}$ & $97,50 \mathrm{a}$ & $92,50 \mathrm{a}$ & $92,50 \mathrm{a}$ \\
\hline Glyphosate + carfentrazone-ethyl & $0,42+0,03$ & $95,00 \mathrm{a}$ & $43,75 \mathrm{~b}$ & $12,50 \mathrm{~cd}$ & $2,50 \mathrm{~b}$ \\
\hline Glyphosate + chlorimuron-ethyl & $0,42+0,15$ & $17,50 \mathrm{~cd}$ & $41,25 \mathrm{~b}$ & $95,75 \mathrm{a}$ & $82,00 \mathrm{~b}$ \\
\hline Glyphosate + metribuzin & $0,42+0,48$ & $97,50 \mathrm{a}$ & $100,00 \mathrm{a}$ & $100,00 \mathrm{a}$ & $100,0 \mathrm{a}$ \\
\hline Glyphosate + sulfentrazone & $0,42+0,6$ & $30,00 \mathrm{c}$ & $12,50 \mathrm{~cd}$ & $13,75 \mathrm{c}$ & $23,75 \mathrm{c}$ \\
\hline Glyphosate + 2,4-D & $0,42+1,0$ & $18,75 \mathrm{~cd}$ & $32,50 \mathrm{bc}$ & $18,75 \mathrm{c}$ & $31,25 \mathrm{c}$ \\
\hline C.V. (\%) & & 31,25 & 19,02 & 17,50 & 15,09 \\
\hline D.M.S. $5 \%$ & & 15,69 & 17,43 & 15,45 & 15,33 \\
\hline $\mathrm{F}$ tratamentos & & $75,13 * *$ & $134,57 * *$ & $181,32 * *$ & $147,98 * *$ \\
\hline
\end{tabular}

Médias na mesma coluna seguidas da mesma letra não diferem entre si, a 5\% de probabilidade pelo teste de Tukey. $* * \mathrm{p} \leq 0,01$.

Os tratamentos sulfentrazone isolado, glyphosate + sulfentrazone e glyphosate $+2,4-$ D não controlaram satisfatoriamente a espécie de $C$. bonariensis aos 35 DAT, uma vez que as plantas apresentaram poucos sinais de fitotoxicidade, sendo que para glyphosate + sulfentrazone, as plantas apresentaram sinais de rebrote.

O estádio de desenvolvimento das plantas de buva no momento da aplicação afeta significativamente a eficiência dos herbicidas glyphosate + 2,4-D. De acordo com Oliveira Neto et al. (2010), excelentes controles desta espécie ocorreram quando as aplicações de glyphosate + 2,4-D foram realizadas com plantas em altura inferior a $6 \mathrm{~cm}$. No presente estudo, as plantas encontravam-se nessa fase, entretanto, a aplicação foi realizada em condições de baixa umidade e alta temperatura, o que pode ter influenciado no resultados de alguns tratamentos. 
Em relação à biomassa seca das plantas, para $C$. bonariensis observou-se que houve aumento da biomassa nos tratamentos de glyphosate+2,4-D (9\%), bentazon (21\%) e chlorimuron-ethyl (17\%), em relação à testemunha. Nos demais tratamentos, houve redução da biomassa seca em relação a testemunha, destacando carfentrazone-ethyl com redução de $92 \%$ e as misturas em tanque de glyphosate + bentazon, glyphosate + chlorimuron - ethyl e glyphosate + metribuzin, onde ocorreram redução da biomassa, de $98 \%$, $81 \%$ e $95 \%$, respectivamente (Tabela 3 ).

Para C. canadensis observa-se que todos os tratamentos com herbicidas aplicados isoladamente não foram eficientes dos 7 aos 35 DAT (Tabela 4). Já em trabalho realizado por Eubank et al. (2008), o amônio-glufosinato aplicado isoladamente em $C$. canadensis, na dose de $0,47 \mathrm{~kg} \mathrm{ha}^{-1}$, resultou em níveis de controle superior a $85 \%$.

Os tratamentos eficazes no controle dessa espécie (> 80\%) foram os aplicados em mistura com glyphosate, destacando: amônioglufosinato $(99,50 \%)$, bentazon $(92,50 \%)$ e metribuzin $(100 \%)$.

O tratamento de glyphosate + carfentrazone-ethyl foi eficiente apenas na primeira avaliação, com controle de $85 \%$. Entretanto, a partir dos 14 DAT o nível de controle foi reduzido para $60 \%$ e aos 35 DAT foi caracterizado como $16 \%$, demonstrando a capacidade de recuperação desta espécie. Shrestha et al. (2008), relataram que carfentrazone-ethyl não controla a $C$. canadensis de maneira efetiva, mesmo sendo aplicado em pós-emergência inicial.

As associações de glyphosate com chlorimuron-ethyl, sulfentrazone e 2,4-D aos 35 DAT, não conseguiram controlar a espécie de maneira satisfatória. Glyphosate + chlorimuron-ethyl proporcionou $92,50 \%$ controlou aos 28 DAT, mas aos 35 DAT as plantas apresentaram sinais de recuperação com rebrote, diminuiu o índice de controle para 75,0\%. Carvalho \& Cavazzana (2000) utilizando chlorimuron-ethyl (10 e $20 \mathrm{~g}$ $\mathrm{ha}^{-1}$ ), aplicado juntamente com o glyphosate reduziu a população de Bidens pilosa durante o ciclo da cultura da soja, porém o mesmo tratamento não teve efeito significativo no controle de Commelina benghalensis, mostrando que o efeito é dependente da espécie.

As plantas tratadas com glyphosate + metribuzin foram completamente controladas aos 28 DAT. Já quando o herbicida metribuzin foi aplicado isolado não apresentou resultado eficaz sobre $C$. canadensis, caracterizando nível de controle de apenas $5,00 \%$.

Houve aumento da biomassa seca nos tratamentos que consistiam nas misturas em tanque de glyphosate com sulfentrazone e 2,4$\mathrm{D}$, assim como no uso isolado de amônioglufosinato, bentazon, carfentrazone, chlorimurom-ethyl, metribuzim e sulfentrazone (Tabela 5). Para carfentrazone-ethyl isolado houve aumento de $45,45 \%$ da biomassa seca, já na mistura de glyphosate + carfentrazone-ethyl ocorreu redução de $9,10 \%$ da biomassa em relação à testemunha. $\mathrm{O}$ aumento da biomassa seca desses tratamentos pode ser justificado pelo baixo nível de controle obtido aos 35 DAT. O mesmo ocorre para bentazon isolado, que aos 35 DAT obteve uma taxa de controle de 5,0\%, ocasionando aumento de 40,19\% na biomassa seca. Já quando aplicado em mistura com glyphosate à taxa de controle foi de $92,50 \%$, ocorrendo redução de 40,19\% na biomassa seca, em relação à testemunha.

A redução da biomassa seca da parte aérea foi constatada nos tratamentos que constituíram das misturas em tanque de glyphosate com amônio-glufosinato, bentazon, carfentrazone-ethyl, chlorimuron-ethyl e metribuzin, as quais foram os tratamentos que obtiveram as maiores taxas de controle aos 35 DAT, com exceção do tratamento de glyphosate + carfentrazone-ethyl, que controle 95\% apenas nos 7 DAT (Tabela 5). 
Tabela 3. Porcentagem da massa seca da parte aérea de C. bonariensis submetida a diferentes tratamentos herbicidas, em relação à testemunha.

\begin{tabular}{lcc}
\hline \multicolumn{1}{c}{ Tratamentos } & $\begin{array}{c}\text { Dose i.a. } \\
\mathrm{kg} \mathrm{ha}^{-1}\end{array}$ & $\begin{array}{c}\text { Massa seca da parte } \\
\text { aérea (\%) }\end{array}$ \\
\hline Testemunha & 0,00 & $100,00 \mathrm{~b}$ \\
Glyphosate & 0,42 & $35,15 \mathrm{c}$ \\
Amônio-glufosinato & 0,50 & $35,71 \mathrm{c}$ \\
Bentazon & 0,72 & $121,00 \mathrm{a}$ \\
Carfentrazone- ethyl & 0,03 & $8,00 \mathrm{~d}$ \\
Chlorimuron-ethyl & 0,15 & $117,00 \mathrm{a}$ \\
Metribuzin & 0,48 & $28,58 \mathrm{c}$ \\
Sulfentrazone & 0,60 & $28,00 \mathrm{c}$ \\
Glyphosate + amônio-glufosinato & $0,42+0,50$ & $21,42 \mathrm{~cd}$ \\
Glyphosate + bentazon & $0,42+0,72$ & $2,00 \mathrm{e}$ \\
Glyphosate + carfentrazone-ethyl & $0,42+0,03$ & $14,29 \mathrm{~d}$ \\
Glyphosate + chlorimuron-ethyl & $0,42+0,15$ & $19,00 \mathrm{~d}$ \\
Glyphosate + metribuzin & $0,42+0,48$ & $5,00 \mathrm{de}$ \\
Glyphosate + sulfentrazone & $0,42+0,60$ & $14,29 \mathrm{~d}$ \\
Glyphosate + 2,4-D & $0,42+1,0$ & $109,00 \mathrm{a}$ \\
\hline C.V. $\%)$ & & 14,17 \\
D.M.S. $5 \%$ & & 15,66 \\
F tratamentos & & $201,63 * *$ \\
\hline Médias na mesma coluna seguidas da mesma letra não diferem entre si, a 5\% de probabilidade pelo teste de Tukey. \\
$* *$ p $\leq 0,01$. & &
\end{tabular}

Tabela 4. Porcentagem de controle de $C$. canadensis aos 7, 14, 28 e 35 DAT.

\begin{tabular}{|c|c|c|c|c|c|}
\hline \multirow{3}{*}{ Tratamentos } & \multirow{3}{*}{$\begin{array}{c}\text { Dose } \\
\text { i.a. kg.ha- }\end{array}$} & \multicolumn{4}{|c|}{ DAT } \\
\hline & & 7 & 14 & 28 & 35 \\
\hline & & \multicolumn{4}{|c|}{$\%$} \\
\hline Testemunha & 0,00 & $0,00 \mathrm{e}$ & $0,00 \mathrm{~h}$ & $0,00 \mathrm{~d}$ & $0,00 \mathrm{~g}$ \\
\hline Glyphosate & 0,42 & $27,50 \mathrm{de}$ & $8,75 \mathrm{fgh}$ & $1,25 \mathrm{~d}$ & $3,75 \mathrm{~g}$ \\
\hline Amônio-glufosinato & 0,50 & 76,25 a & $78,75 \mathrm{~b}$ & $36,25 \mathrm{~b}$ & $22,50 \mathrm{e}$ \\
\hline Bentazon & 0,72 & $27,50 \mathrm{de}$ & $1,25 \mathrm{gh}$ & $0,00 \mathrm{~d}$ & $5,00 \mathrm{~g}$ \\
\hline Carfentrazone- ethyl & 0,03 & $66,25 \mathrm{~b}$ & $30,25 \mathrm{e}$ & $5,00 \mathrm{~d}$ & $1,25 \mathrm{~g}$ \\
\hline Chlorimuron-ethyl & 0,15 & $7,50 \mathrm{e}$ & $8,75 \mathrm{f}$ & $0,00 \mathrm{~d}$ & $3,75 \mathrm{~g}$ \\
\hline Metribuzin & 0,48 & $22,50 \mathrm{de}$ & $1,25 \mathrm{gh}$ & $2,50 \mathrm{~d}$ & $5,00 \mathrm{~g}$ \\
\hline Sulfentrazone & 0,6 & $11,25 \mathrm{e}$ & $13,75 \mathrm{f}$ & $17,50 \mathrm{c}$ & $13,75 \mathrm{f}$ \\
\hline Glyphosate + amônio-glufosinato & $0,42+0,5$ & $92,50 \mathrm{a}$ & $100,00 \mathrm{a}$ & $100,00 \mathrm{a}$ & $99,50 \mathrm{a}$ \\
\hline Glyphosate + bentazon & $0,42+0,72$ & $87,50 \mathrm{a}$ & $91,25 \mathrm{ab}$ & $92,50 \mathrm{a}$ & $92,50 \mathrm{~b}$ \\
\hline Glyphosate + carfentrazone-ethyl & $0,42+0,03$ & $85,00 \mathrm{a}$ & $60,00 \mathrm{c}$ & $15,00 \mathrm{c}$ & $16,25 \mathrm{e}$ \\
\hline Glyphosate + chlorimuron-ethyl & $0,42+0,15$ & $31,25 \mathrm{bc}$ & $45,00 \mathrm{~d}$ & $92,50 \mathrm{a}$ & $75,00 \mathrm{c}$ \\
\hline Glyphosate + metribuzin & $0,42+0,48$ & $85,00 \mathrm{a}$ & $97,50 \mathrm{a}$ & $100,0 \mathrm{a}$ & $100,0 \mathrm{a}$ \\
\hline Glyphosate + sulfentrazone & $0,42+0,6$ & $16,25 \mathrm{e}$ & $10,00 \mathrm{fg}$ & $2,50 \mathrm{~d}$ & $23,75 \mathrm{e}$ \\
\hline Glyphosate + 2,4-D & $0,42+1,0$ & $43,75 \mathrm{~b}$ & $58,75 \mathrm{c}$ & $31,25 \mathrm{~b}$ & $32,50 \mathrm{~d}$ \\
\hline C.V. $(\%)$ & & 37,23 & 10,15 & 9,39 & 19,59 \\
\hline D.M.S. $5 \%$ & & 18,21 & 9,21 & 7,35 & 8,96 \\
\hline F tratamentos & & $55,56 * *$ & $434,62 * *$ & $702,29 * *$ & $585,83 * *$ \\
\hline
\end{tabular}

Médias na mesma coluna seguidas da mesma letra não diferem entre si, a 5\% de probabilidade pelo teste de Tukey. $* * \mathrm{p} \leq 0,01$. 
Tabela 5. Porcentagem da massa seca da parte aérea de C. canadensis submetida a diferentes tratamentos herbicidas, em relação à testemunha.

\begin{tabular}{lcc}
\hline \multicolumn{1}{c}{ Tratamentos } & $\begin{array}{c}\text { Dose i.a. } \\
\mathrm{kg} \mathrm{ha}^{-1}\end{array}$ & $\begin{array}{c}\text { Massa seca da parte } \\
\text { aérea (\%) }\end{array}$ \\
\hline Testemunha & 0,00 & $100,00 \mathrm{bc}$ \\
Glyphosate & 0,42 & $90,90 \mathrm{c}$ \\
Amônio-glufosinato & 0,50 & $109,09 \mathrm{~b}$ \\
Bentazon & 0,72 & $136,36 \mathrm{a}$ \\
Carfentrazone- ethyl & 0,03 & $145,45 \mathrm{a}$ \\
Chlorimuron-ethyl & 0,15 & $136,36 \mathrm{a}$ \\
Metribuzin & 0,48 & $127,27 \mathrm{ab}$ \\
Sulfentrazone & 0,60 & $100,00 \mathrm{bc}$ \\
Glyphosate + amônio-glufosinato & $0,42+0,50$ & $9,09 \mathrm{f}$ \\
Glyphosate + bentazon & $0,42+0,72$ & $54,54 \mathrm{~d}$ \\
Glyphosate + carfentrazone-ethyl & $0,42+0,03$ & $90,90 \mathrm{c}$ \\
Glyphosate + chlorimuron-ethyl & $0,42+0,15$ & $63,63 \mathrm{~d}$ \\
Glyphosate + metribuzin & $0,42+0,48$ & $27,27 \mathrm{ef}$ \\
Glyphosate + sulfentrazone & $0,42+0,60$ & $118,18 \mathrm{~b}$ \\
Glyphosate + 2,4-D & $0,42+1,0$ & $127,27 \mathrm{ab}$ \\
\hline C.V. $\%$ (\%) & & 10,08 \\
D.M.S. $5 \%$ & & 24,40 \\
F tratamentos & & $76,69 * *$ \\
\hline Medis.
\end{tabular}

Médias na mesma coluna seguidas da mesma letra não diferem entre si, a 5\% de probabilidade pelo teste de Tukey. $* * \mathrm{p} \leq 0,01$.

\section{Conclusões}

As espécies $C$. bonariensis e $C$. canadensis apresentam folhas anfiestomáticas e estômatos classificados como anomocíticos, sendo que ambas possuem tricomas tectores unicelulares e pluricelulares, assim como ausência de cristais de ceras epicuticulares.

Para $C$. bonariensis os tratamentos mais efetivos foram amônio - glufosinato isolado, e as misturas em tanque de glyphosate com amônio glufosinato, bentazon, chlorimuronethyl e metribuzin. Para C. canadensis, os tratamentos mais efetivos foram às misturas de glyphosate com amônio-glufosinato, bentazon e metribuzin.

\section{Referências}

\section{ALAM}

LATINOAMERICANA Recomendaciones sobre unificación de los sistemas de evaluación em ensayos de control de malezas. ALAM, Bogotá, v.1, p.35-38, 1974.
APPEZZATO-DA-GLORIA, B.; GUERREIRO, C.S.M. Anatomia vegetal. Viçosa: Universidade Federal de Viçosa, 2003. 438p.

BAKER, E.A.; BUKOVAC, M.J. Characterization of the components of plant cuticles in relation to the penetration of 2,4D. Annals of Applied Biology, v.67, n.3, p.243-253, 1971.

BUKOVAC, M.J., PETRACEK, P.D. Characterizing pesticide and surfactant penetration with isolated plant cuticles. Pesticide Science, v.37, n.2, p.179-194, 1993.

CARVALHO, F.T.; CAVAZZANA, M.A. Eficácia de herbicidas no manejo de plantas daninhas para o plantio direto de soja. Revista Brasileira de Herbicida, v.1, n.2, p.167-172, 2000.

EUBANK, T.W. et al. Glyphosate-resistant horseweed (Conyza canadensis) control using glyphosate-, paraquat-, and glufosinate-based herbicide programs. Weed Technology, v.22, n.1, p.16-21, 2008. 
FERREIRA, E. et al. Estudos anatômicos de folhas de plantas daninhas: I - Nicandra physaloides, Solanum viarum, Solanum americanum e Raphanus raphanistrum. Planta Daninha, v. 20, n. 2, p. 159-167, 2002.

OLIVEIRA NETO, A.M. et al. Manejo de Conyza bonariensis com glyphosate $+2,4-\mathrm{D}$ e amônio-glufosinate em função do estádio de desenvolvimento. Revista Brasileira de Herbicidas, v.9, n.3, p.73-80, 2010.

KISSMANN, K.G.; GROTH, D. Plantas infestantes e nocivas. 2. ed. São Bernardo do Campo: Basf., 1999 p. 152-284.

LAMEGO, F.P.; VIDAL, R.A. Resistência ao glyphosate em biótipos de Conyza bonariensis e Conyza canadensis no estado do Rio Grande do Sul, Brasil. Planta Daninha, v.26, n.2, p.467-471, 2008.

LARCHER, W. Ecofisiologia vegetal. RiMa, São Carlos, 2004, 531p.

LAZARATO, C.A. et al. Biologia e ecologia de buva ( $C$. canadensis e $C$. bonariensis). Ciência Rural, v. 38, n. 3, p. 852-860, 2008.

MONQUERO, P.A. et al. Glyphosate em mistura com herbicidas alternativos para o manejo de plantas daninhas. Planta Daninha, v. 19, n.3, p. 375-380, 2001.

MONQUERO, P.A. Dinâmica populacional e mecanismos de tolerância de espécies de plantas daninhas ao herbicida glyphosate. 2003. 99 f. Tese (Doutorado em Fitotecnia) Escola Superior de Agricultura "Luiz de Queiroz", Piracicaba, 2003.

MOREIRA, M.S. et al. Resistência de Conyza canadensis e $C$. bonariensis ao herbicida glyphosate. Planta Daninha, v.25, n.1, p.157164, 2007.

OLIVEIRA JUNIOR, R. S.; BACARIN, M. A. Absorção e translocação de herbicidas. In: OLIVEIRA JUNIOR, R. S.; CONSTANTIN, J. (Coords.). Plantas daninhas e seu manejo. Guaíba: Agropecuária, 2001. p. 261-290.
PAULA, J.M. et al. Manejo de Conyza bonariensis resistente ao herbicida glyphosate. Planta Daninha, v.29, n.1, p.217-227, 2011.

PROCÓPIO, S.O. et al. Estudos anatômicos de folhas de espécies de plantas daninhas de grande ocorrência no Brasil III-Galinsoga parviflora, Crotalaria incana, Conyza bonariensis e Ipomoea cairica. Planta Daninha, v.21, n.1, p.1-9, 2003 b.

PROCÓPIO, S.O. et al.Anatomia foliar de plantas daninhas do Brasil. Viçosa: UFV, 2003a.

SHRESTHA, A. et al. Biology and management of Horseweed and Hairy Fleabane in California. University of California, California, publication 8314, 2008. Disponivel em: <http://ucanr.org/freepubs/docs/8314.pdf $>$.

Acesso em: 01 ago. 2010.

TANAKA, F.A.O; KITAJIMA, E. W. Treinamento em técnicas de microscopia eletrônica de varredura. Piracicaba: Apostila do curso, 2009. 100p.

VARGAS, L. et al. Buva (Conyza bonariensis) resistentes ao glyphosate na região sul do Brasil. Planta Daninha, v.25, n.3, p.573-578, 2007

VIDAL, R.A.; TREZZI, M.; LOUX, M. Definindo resistência aos herbicidas. Revista Plantio Direto, v.100, n.4, p.18-19, 2007.

WERTH, J. et al. Applying the double knock technique to control Conyza bonariensis. Weed Biology And Management, p. 01-08, 2010. Disponível em: <http://onlinelibrary.wiley.com.ez31.periodico s.capes.gov.br/doi/10.1111/j.1445-

6664.2010.00360.x/full>. Acesso em: 25 ago. 2011.

YAMAUTI, M.S. et al. Controle químico de biótipos de buva (Conyza canadensis e Conyza bonariensis) resistentes ao glyphosate. Revista Ciência Agronômica, v.41, n.3, p.495-500, 2010. 